\title{
Verzeichnis der Mitarbeiter und ihrer Beiträge in den Indogermanischen Forschungen Band 1-40.
}

Ammann, H. Die Stellungstypen des lateinischen attributiven Adjektirums und ihre Bedeutung für die Psychologie der Wortstellung auf Grund von Ciceros Briefen an Attikus untersucht 29, 1.

Ascoli, G. J. Lat. rīd- (ridere), idg. vrizd-13,278.

Back, R. Medizinisch-Sprachliches 40,162.

Bahder, K. v. Die e-Abstoßung bei dem nhd. Nomen 4,352. - Zur hochdeutschen Lautlehro (hd. $f$ $=$ wgerm. b) 14,258.

Bang, W. Altpersisches 8,291.

Bartholomae, Chr. Arica I 1,178. - Griech.óvoua >óvóuaros $1,300$. - Arica II 1,486. - Arica III 2 , 260. - Zur Vokaldehnung im Präteritum 3,1. - Arica IV 3,100. - Zur l-Frage 3,157. - Arica V 4,121. - Arica VI 5,215. Arica VII 5,355. - Zum Cippus Abellanus 6,307. - Die neunte Präsensklasse der Inder 7,50. Idg. $e+$ Nasal im Tiefton 7,82. Arica VIII 7,223. - Arica IX 8,229. - Arica X 9,2,2. Arica XI 10,1. - Arica XII 10, 189. - Arica XIII 11,113. Arica XIV 12,93. - Arica XV 21,347. - ZurUmschreibung der arischen Sprachen 21,366. - Zu den arischen Wörtern für 'der erste' und 'der zweite' 22,95. Fortsetzung 23,43. - Fortsetzung 23,318. - Arica XVI 25,167. Der indogermanische Name der Plejaden 31,35. - Berichtigung dazu 32,413. - Wandersprüche im Mittelpersischen 37,87. - Arica XVII 38, 1 - Arica XVIII 38, 26.
Baudiě, J. Das slavische Imperfektum 23, 135 .

Baunack, J. Zu Inschriften aus Troezen 4,187.

Baunack, Th. Rv. X. 40. 3. prātár jarethe jaranéva kápayā 8,278.

Behaghel, O. Die Herstellung der syntaktischen Ruhelage im Deutschen 14,438. - Zur Etymologie von $\operatorname{man}$ 'nur' 22,340. - Beziehungen $z$ wischen Umfang und Reihenfolge von Satzgliedern 25, 110. - Fernstellung zusammengehöriger Wörter im Deutschen 31,377. - Die 2. Pers. Sing. Ind. Prät. st. Flexion im Westgermanischen 40,167.

Bender, H. H. Notes on Indo-Iranian -vant 30,137. - The Accent of Sanskrit -mant and -vant 34, 383.

Bergman, G. Lat. pi $c a, p \bar{\imath} c u s$ 39,105.

Berneker, E. Zur Präsensflexion der lateinischen primäron $i o-V e r b a ~ 8$, 197. - Etymologisches 8, 283. Zur germanischen Verbalflexion 9,355. - Etymologisches 9,360. - Von der Vortretung des idg ě $u$ im baltisch-slavischen Sprachzweig 10,145. - Kyrills Übersetzungskunst 31,399.

Björkman, E. Neuschwed. gosse 'Knabe, Junge', eine semasiologisch-methodologische Studie 30,252 .

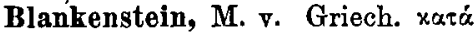
und seine Verwandten 21,99. Etymologien 23,131.

Bloch, J. Tamoul väddyar' sanskrit upādhyāya 25,239.

Bloomfield, $M$. On the so-called root-determinatives in the IndoEuropean languages' 4,66. - On 
some disguised forms of Sanscrit paśu 'cattle' 25,185. - On the variable position of the finite verb in oldest Sanskrit 31,156.

Blưlmel, R. Der Ursprung des griechischen Bereichsakkusativs und anderes $33,1 .-{ }^{-}$Verbindung von Ganzem und Teil ' und andereGruppen syntaktischer Beziebungen in der nhd. Umgangssprache 34,285 .

Böhtlingk, O. Die 1. Pers. Sing. Med. des umschriebenen Futurs im Sanskrit 6,342. - Sprachliche Minutien 7,270.

Bojunga, $\mathbf{K}$. Der idg. Konjunktiv im Germanischen 2,184.

Bradke, P. v. Zwei sprachgeschichtliche Skizzeu 4,85. - Über die sanskritische Form der Wurzeln auf skr. -áni und -ámi (mit dem 'Bindevokal' $i$ ) vor einem Konsonanten, wenn die Wurzel den Akzent verloren hat 5,266. - Ǘber den 'Bindevokal' skr. $i$ griech. $\alpha$ (lat. $i$ got. $u$ ) im Perfektum 8,123.

Braune, W. Germanisches ss und die Hessen 4,341.

Brännlich, A. F. A theory of the origin of hypotaxis 35,237.

Bremer, O. Der germanische Himmelsgott 3,301. - Relative Sprachchronologie 4,8. - Die germanische 'Brechung' 26, 148.

Brenner, O. Ein Fall von Ausgleichung des Silbengewichts in bairischen Mundarten 3,297. Zur Ausgleichung des Silbengewichts 5,345. - Urgerm. $a$ in unbetonter Silbe 14,363.

Brïch, J. Nhd. Attich 39,122, Lateinische Etymologien (I. Über einige Namen des Wacholders. II. Über lat. brisa) $\mathbf{4 0 , 1 9 6 .}$

Brilckner, A. Über etymologische Anarchie 23, 206.

Brugmann, K. und W. Streitberg, Zu Franz Bopps hundertjährigem Geburtstage 1,5. - Lat. velimus got. wileima und ags. eard 1,81 .

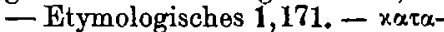

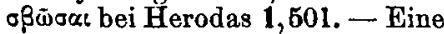
archaische Weihinschrift aus Ker-

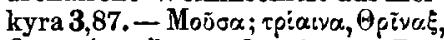
Opivari perf. sē $\bar{d}$ 3,303. - Latina: 1. acer ācris äcre. 2. nullus est, quīn 4, 218. - Die mit dem Suffix -to- gebildeten Partizipia im Verbalsystem des Lateinischen und des Umbrisch-Oskischen. Fine syntaktische Untersuchung 5,89. Griech. xทุp 5,341. - Nhd. koth 5,375. - Ahd. sibun und äband 5,376. - 'Apiáov 5,379. - Die lat. Partikel ne ('nicht') in $\mathrm{Zu}$ sammensetzung mit vokalisch anlautenden Wörtern 6,79. - Der präteritale Bildungstypus ahd. hiaz aisl. hét und ahd. liof aisl. hliop 6,89 - Die Verbindung dentaler Verschlußlaut $+s+t$ im Lateinischen und im Germanischen 6, 102. - Zur Transskriptionsmisère 7, 167. - Lat. id meà rēfert 8,218. Dissimilatorische Verändßrungen von $\bar{e}$ im Griechischen und Aristarchs Regel über den homerischen Wechsel von $\eta$ und $\varepsilon \iota$ vor. Vokalen 9,153. - Die sogen. unechten Diphthonge $\varepsilon$ : und oo 9,343. - Lateinische Etymologien 9,346. -- Lat. multi-angulus 9,354. - Die Herkunft der griech. Substantiva auf

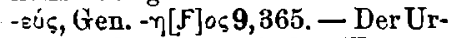
sprung der germanischen Kompa-

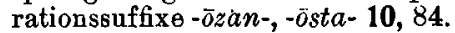
- Griechische und italische Miszellen 11,99. - Aksl. zupa 'Bezirk' 11,111. - Zur griechischen und lateinischen Etymologie u. Stammbildungslehre 11,266. - Der indoiranische Feminintypus $n d \vec{d} r-\bar{\imath} 12,1$.

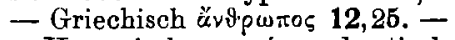
- Homerisch pevo:váw und gotisch briggan, zwei Fälle von Wurzelangleichung 12,150. - Lateinisch vicissim 12,181. - Nochmals lat. aliēnus, laniēna 12,389. - Lat. dèierā̀e, perierāre peiierāre, èierūre und aerumna 12,396. - Lateinisch cedo und arcesso, incesso 13,84. - Wortgeschichtliche Miszellen 13,144. - Ahd. frist und got. frisahts 13,164. - Die ionischen Iterativpräterita auf -oxov

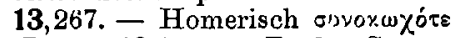
(B 218) 13,280. -- Zu den Superlativbildungen des Griechischen und desLateinischen 14,1.-Griech. xpoxóṡtios 15,8. - Altitalisches 15,69. - Beiträge zur griechischen, germanischen und slavischen Wort. forschung 15,87. - Zur griech. und germ. Präsensflexion 15, 126 . 
- Slavisch $l i$ und lettisch lái 15 , 339. - Etymologische Miszellen 16,491. - Umbr. persnihimu und die altind. neunte Präsensklasse 16,509. - 'Exwiv und seine griechischenVerwandten 17,1, - Lat. humānus 17,166. - 'Axрй $\pi \varepsilon \delta 05$ (Nachtrag zu S. 8) 17, 174. - Zur Bildung der 2. Pers. Sing. Akt. in den indogermanischen, insbesondere den baltischen Sprachen 17, 177. - Griech. Èvta.viós und got. wis 17,319. - Verdunkelte Nominalkomposita des Griechischen und des Lateinischen 17,351. Griech. vís viós víwós und ai. sūnús got. sunus 17,483. - Lat. annus osk.-umbr. akno- got. apna17,492. - Zur Wortzusammensetzung in den idg. Sprachen (1. Die Stellung der Bahuvrihi im Kreis der Nominalkomposita. 2. Der àxér.axos-Typus und Verwandtes) 18,59. - Der Kompositionstypus

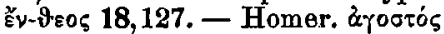
und ărpך 18,129. - Varia 18, 381. - Alte Wortdeutungen in neuer Beleuchtung 18,423. - Umbrisch purditom 18, ā31. - Griechische Miszellen 19,212. - Das Genus der Deminutivbildungen 19,215. Zu den Benennungen der Personen des dienenden Standes in den indogermanischen Sprachen 19,377. Griech. b̀ otpós 19,399. - Die lit. Verbalabstrakta auf-imas 19,400.

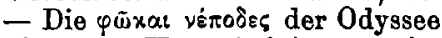

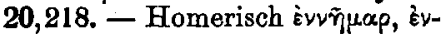

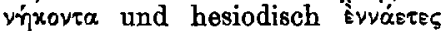
20,225. - Griechisch \$̛́ćs, Ẽ, , dós 20, 363. - Setzung und Nichtsetzung des Zahlworts Eins zu Quantitätssubstantiva in den idg. Sprachen 21,1. - Nochmals lat. rêfert 21,200. - Noós, nurus, snußd und die die griechischen und italischen femininen Substantiva auf -os 21, 315. - Zur haplologischen Wortkürzung 21,367. - Die Anomalien in der Flexion von griech. roví, armen. kin und altnord. kona 22, 171. - Griechisch '̌vos und övos 22,197. - Der slav. Instr. Plur. auf $-y$ und der aw. Instr. Plur. auf -ǔs 22,336. - Zur Frage der Einführung einer künstlichen internationalen Hilfssprache 22,365. -
Die lateinischen Akkusative $m \bar{e}(d)$, $t \bar{e}(d), s \bar{e}(d) 23,311 .-{ }^{\prime} \mathrm{H} i \pi \pi 05^{\circ} \mathrm{Die}$ Reiterei' und Verwandtes 24,62. Altitalische Miszellen 24,72. - Zur lateinischen Wortforschung 24, 158. - Gotisch bairōs und der Dual der Indogermanen 24,165.

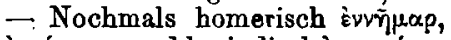

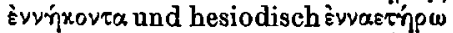
24,307. - Nachtrag zu 24, $165 \mathrm{ff}$. 24,314. - Der sogenannte Akkusativ der Beziehung im Arischen, Griechischen, Lateinischen, Germanischen 27, 121. - Der lat. Ablativus comparationis 27,159. Adverbia aus dem maskulinischen Nominativus Singularis prädikatives Adjektiva 27, 233. - Wortgeschich tliche Miszellen 28,285. Griechische und lateinische Etymologien 28,355. - Lateinisch fuère, fuērunt, fuërunt 28, 379. Zur griechischen und italischen Wortforschung (1. Griech. $\gamma \tilde{\eta}$, үaia,

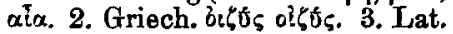
imus) 29,200. - Verdunkelte Präpositionalkomposita im Griechischen 29,229. - Zu den Imperativendungen im Umbrischen 29,243. - Die litauischen Imperative anf

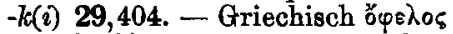

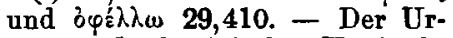
sprung des lateinischen Konjunktivus Imperfekti und Konjunktivus Plusquamperfekti 30,338. - Wortgeschichtliche Miszellen 30,371. Zu den reduplizierten Verbalbildungen im Indoiranischen 31,89. - Alpéw 32, 1. - Vedisch irajyáti, iradhanta und Verwandtes 32,58.

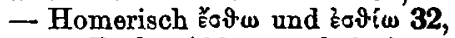
63. - Zu den Ablautsverhältnissen der sogenannten starken Verba des Germanischen 32,179. - Griechisch $\alpha \pi \tau \omega$ und seine außergriechischen Verwandten 32,319. Abkürzung im sprachlichen Ausdruck, ihre Anlässe und ihreGrenze 32,368. - Die gotische Partikel $-u h,-h$ 33,173. - Der gotische Genitivus Pluralis auf $-\bar{e} 33,272$. - Gotisch usstagg 'stich aus' 33, 284. - Zur nominalen Stammbildung der germanischen Sprachen 33,300. - Die griechischen Desiderativa anf -osicur nebst xeicur 33,332. - Die Entstehung des alt- 
indischen Prekativs 34, 392. - Zur Geschichte der lateinischen Nomina mit Formans -ti- 34,397. Zwei oskische Adverbialbildungen (1. ekss, ex. 2. púkappéd [p]ocapid pocapit) 34,402. - Úber einige $z u$ ovivnue gehörige Nominalformen 35,94. - $-\bar{a} i \mathrm{im}$ altindischen Konj. Medii 36,164. - Lat. aemulus, aequos, imitāari, imāgo, griech. a $\psi^{\mathbf{k}} \psi \alpha$, aimís, got. ibns 37,155, - Griech.

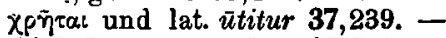
Ahd. henna, ags. hen 37,249. Gleichklangvermeidung in der lautgesetzlichen Entwicklung und in der Wortbildung 38,117. - Zur griechischen und lateinischen Wortgeschichte 38,128. - Haplologisches im heutigen Rheinfränkischen 38,206. - Das got. -adaPassivum 39,26. - Zur Geschichte des Ausrufungssatzes im Griechischen 39,114. - Dissimilation bei garderobe und parterre 39,130 . Zur Frage des Ursprungs der Personalendungen des indogermanischen Verbums 39,131. - Griech. $\sigma:-$ als sinnverstärkendes Vorderglied in Nominalkomposita 39, 140.

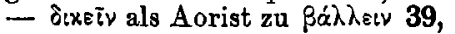
144. - Böot. $\pi \tau \varepsilon \varepsilon \dot{\omega} \omega$ 39,149. Altlat.humus Gen. Sing. = griech. $\chi$ Эrovós 39,151. - Lat. sevērus 39, 154. - Analogische Neuerung in den Ausgängen der Formen des Verbum finitum in den idg. Sprachen 39,157 .

Brugmann, 0. Andes - Andicus 24,128.

Buck, C. D. Do the sounds of the new guttural series (or the nonlabialized velars) suffer dentalization in Greek? 4,152. - Critical notes to Oscan Inscriptions 12,13. - Greek Notes 25,257.

Bugge, S. Beiträge zur etymologischen Erläuterung der armenischen Sprache 1,437. - Über den Einfluß der armenischen Sprache auf die gotische 5,168. - Nachtrag dazu 5,y74. - Einige Zahlwörter im Lykischen 10,59.

Bülbring. K.H. Vokativformen im Altenglischen 6,140.

Bulitsch, S. Slavische Miszellen 5,389 .

Burchardi, G. Eine niederdeutsche Indogermanische Forsehungen XL.
Form, die es gar nicht gibt! 38, 200. - "Halb sieben sein'='betrunken sein' 38,201. - Nachträge $38,205$.

Buttenwieser, M. Zur Geschichte des böotischen Dialekts 28,1.

Caland, W. Syntaktisch-exegetische Miszellen 31,105.

Cappeller, C. Zwölf Pasakos aus dem preußischen Südlitauen 31,427. Noch zwölf Pasakos 35,114.

Chadwick, H. M. Ablaut-Problems in the Idg. Verb. 11,145.

Charpentier,J. Kleine Beiträge zur armenischen Wortkunde $\mathbf{2 5 , 2 4 1 . ~}$ -A westische Etymologien 28,153. - Beiträge zur alt- und mittelindischen Wortkunde 28,157 . Fortsetzung und Schluß 29,367. Die altindischen Perfektformen des Typus daddu 32,92. - Zwei mittelindische Desiderativbildungen 35, 217. - Indische Wörter mit den Endungen -amba-, -imba-, -umba35, 246 .

Ciardi-Dupré, G. Fruchtbäume und Baumfrüchte in den indogermanischen Sprachen 25, 155.

Collitz, H. Bemerkungen zum schwachen Präteritum (1. Zu den Plural- und Optativendungen. 2. $\mathrm{Zu}$ den eigenartigen Endungen des Alemannischen) 34,209.

Compernass, J. Vulgärlateinisches (1. mactāre "schlagen, prügeln", 2. nihilominus 'gleichfalls, ebenso ') 34,389. - Vulgärlatein (1.Part. statt Verb. fin. 2. Ersatz bezw. Umschreibung des präd. Dat. 3. Pro eo ut, pro eo quod) 35,220 .

Conway, R. S. On the change of $d$ to $l$ in Italic 2,157. - Oskisch eituns 3,85. - Minutiae Italicae 4,213.

Cosijn, P. J. Die substantivierten Partizipia präsentia des Urgermanischen 10,112. - Nachtrag dazu 11,204.

Courtenay, J. B. de. Einiges über Palatalisic rung und Entpalatalisierung 4,45. - Zum altkirchenslavischen ojomins 21,196. - Zur 'Sonanten'-Frage 25,77.

Crusins, 0. Über einige mythische Beinamen und Namen derGriechen 4, 169 . 


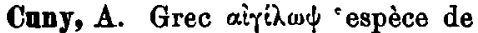
chếne', latin îlex 26,21.

Danielsson, O.A Griech. 2oó́ $\lambda \omega \tau \circ \varsigma$, $\lambda \omega \tau i \varsigma$ 4,158, - Zur i-Epenthese im Griechischen 14,375. - Zur Lehre vom homerischen Digamma 25,264. - Zu einer arkadischen Verbalform 35,99.

Debrunner, A. Za den konsonantischen io-Präsentien im Griechischen 21,13. - SchluB dazu 21, 201. - Die Adjektiva auf - $x \lambda_{\varepsilon \circ \zeta}$ 23,1. - Homerica I 39,202. Homerica II 40,107.

Dekawalles, N. A. Ein hesychisches Fachwort für 'onomatopoëtisches Gebilde' 35, 147.

Delbrück, B. Der Typus $\varphi$ épw yopéw im Arischen 4,132. - $\Phi$ źploros und Verwandtes 14,46. - Das gotische $d u$ und das westgermanische Gerundium 21,355. - Das schwache Adjektivum und der Artikel im Germanischen 26,187.

Diels, H. Griech. xpoxódè os 15,1.

Dieterich, K. Akzent und Bedeutungsverschiebung im Mittel- und Neugriechischen 16,1. - Die präpositionalen Präfixe in der griechischen Sprachentwicklung mit besondererBerücksichtigung des Mittel- und Neugriechischen (Erstes Kapitel : ¿ajó) 24,87.

Dittrich, O. Konkordanz und Diskordanz in der Sprachbildung 25,1.

Edgerton, F. Three Atharva-Veda words in suffixal $-k a 24,291$.

Ehrlich, H. Über die sekundüro Aktivendung der 3. Person Pluralis im Oskisch-Ümbrischen 11,299. Nachträge dazu 11,343.

Endzelin, J.' Zu den kurischen Bestandteilen des Lettischen 33,96. - Weiteres zu den lettischen Intonationen 33,104. - Miszellen 33, 119 .

Ernout, A. Note sur les thèmes en - $u$ - latins 26,91.

Eulenburg, $\mathrm{K}$. Zur Vokalkontraktion im ionisch-attischen Dialekt 15,12y. - Zum Wandel des idg. 0 im Germanischen 16,35.

Fay, E. W. Latin mille again 11,

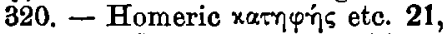
193. - aipwr and imago 26,27. - Is Greek -oúrn cognate with Sanskrit -tvana-m? 29,413. - Ety- mologica 32,330. - Word-Studies 33, 351 .

FoJ, W. Die idg. $s$-Laute ( $s$ und $z$ ) im Keltischen 6,313. - Zur Geschichte des idg. $s$ im Keltischen 8,200. - Ai. káprt : griech. xámpos usw. 8,295. - Zur Syntax von ai. nāma, av. nąma, ap. nāmă usw. $12,172$.

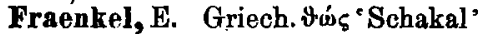
22,396. - Grammatisches und Syntaktisches. (I. Zur Verblassung der einem Worte ursprünglich innewohnenden Spezialbedeutung. II. Behandlung von ersten Kompositionsgliedern als selbständige Nomina., III. Fälle von patronymischem Genetiv statt des zu erwartenden Adjektivs in den äolischen Dialekten. IV. o 83 ś nach dem Komparativ im Sinne von V. Spuren des heimatlichen Dialekts in den hipokratischen Schriften. VI. Eine neue suffixlose 2. sing. imperat. eines urspr. athem. flekt. griech. Verb. VII. Zum dor. Reflexivum. VIII. Zur litauischen Partizipialkonstruktion. IX.Nachtrag zu $\pi \rho s ́ \sigma \beta \varepsilon: \rho \alpha$ und zu delisch

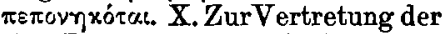
silb. Liqu. in dem "sïdachäischen" Dial.) 28,219. - Zur metaphori-

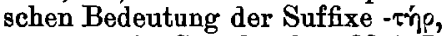

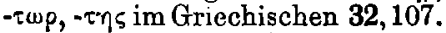

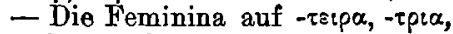

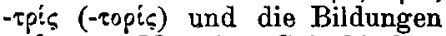

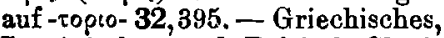
Lateinisches und Baltisch-Slavisches (I. Zu griechischen Inschriften. II. Zu lat. fustis) $\mathbf{4 0 , 8 1}$.

Friedrich, J. Altitalisches (1. Oskisch iiv. 2. Ein faliskischer Saturnier) 37, 141. - Die altpersische Stelle in Aristophanes 'A charnern" (V. 100) 39,93. - KapKrów und Carthago 39,102. - Nachträge dazu 39,231.

Fnnck, A. Lat. prodigium 2,367.

Gabelentz, G. v. d. Hypologie der Sprachen, eine neue Aufgabe der Linguistik 4,1.

Garnier, K. v. com- als perfektivierendes Präfix bei Plautus, samim Rigveda, ouv-bei Homer 25,86.

Ganthiot, R. A propos des nominatifs pluriels lituaniens en $-a \tilde{2}$ 26, 353 . 
Geiger, W. Das afghānische Präteritum 3,111.

Geisan, J.v. Syntaktische Gräzismen bei Apulejus 36,70. - Fortsetzung und SchluB 36,242.

Goetz, G. Spïuchliche Bemerkungen zu Varro de re rustica 31,298.

Grammont, M. Grec rouvós 'nu' 25,371 .

Gray, L. H. Zur indogermanischen Syntax von *nāman 11,307.

Grienberger, v. ZurDuenosinschrift 11,343. - Zur Duenosinschrift 16,27. - Múspell 16,40. - Das carmen aruale 19,140. - Opeinod devincam ted! 21,362. - Die Inschrift der Fuciner Bronze 23,337. - Die Fragmente saliarischer Verse bei Varro und Scaurus 27, 199. - Zur Inschrift des Cippus vom Forum Romanum $130,210$. - Die altlateinische Inschrift von Lucera 33,285. - Zur Inschrift des Cippus vom Forum Romanum 2 37, 122. - Ortsnamenmaterial und Sprachvergleichung 40,135.

Güntert, H. Zur Geschichte der griechischen Gradationsbildungen 27,1. - Zur Bildung der altindischen Desiderativa 30,80. - Ein verkanntes ai. Kompositum 32,102. - Zur' etymologischen Herkunft von lat. haurīe 32,386. - Zur o-Abtönung in den indogermanischen Sprachen 37,1. - Aus der Geschichte einer Negation 40,186.

Gtinther, R. Die Präpositionen in den griechischen Dialektinschriften 20,1. - Die ursprüngliche Gestalt des lateinischen Präverbs re, red 26,94. - $\mathrm{Zu}$ den dorischen Infinitivendungen 32,372. - Griechische Miszellen (I. Die Herkunft des äolischen Optativs. II. Gorty. nisch $\tau$ íi(vs) 33,407.

Hale, W. G. Origin of the distinction of tenses in Latin prohibitions 31,272.

Hatzidakis, G. Ikarisches 2,371. -

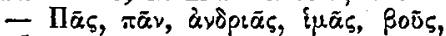

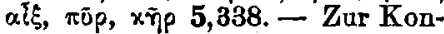
traktion von $\varepsilon \alpha$ nach $\rho$ im Attischen 5,393. - Zur Ethnologie der alten Makedonier 11,313. - Zum Gebrauch der verbalen Medialformen im Neugriechischen 25,357. -
Analogiebildungen im pontischen

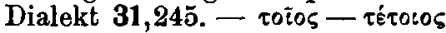
und Verwandtes 32, 352. - Zum neumegarischen Dialekt 36, 287. Alte Buntheit im Neugriechischen 36, 299.

Havers, W. Das Pronomen der JenerDeixis im Griechischen 19,1. Zur Etymologie von griech. $\varphi \alpha \dot{\rho}-$ ursxoy 25,370. - Wortgeschichtliches (Lat. est sub alapa [Petron. Cen.Trim. c. 38]. — Griech. WE Yety.

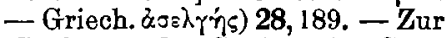
'Spaltung' des Genitivs im Griechischen 31,230. - Abruptes $\tau \alpha \tilde{u} \tau \alpha$ und Verwandtes 32, 150.

Heckmann, J. Über präpositionslose Ortsbezeichnung im Altlateinischen 18,296.

Heilig, O. Hebels 'Hansund Verene' 14,39 .

Heinertz, N. O. Friesisches (1. Die haupttonigen Vokale im Wortauslaut und Hiatus im Altfriesischen. 2. Zur Entwicklung des urg. ai im Friesischen. 3. Zur Entwicklung von urg. $a+\ddot{j} j$ im Friesischen) 30,303. - Friesisches (4. Beiträge zur altfries. Wortkunde) 35,304.

Heinsius, J. Über die Repräsentation von idg. skh im Griechischen 12,178 .

Helm, K. Die Heimat der Germanen und das Meer 24,221.

Helten, W. van. Zur as. Grammatik 5,182. - Weiteres zur as. Grammatik 5,347. - Zum Vokalismus und Konsonantismus der friesischen Dialekte 7,312. - Zur gotischen Grammatik 14,60. - Zur Entwicklung der germ. Komparativ- und Superlativ-Suffixe 16,63. - Zum germanischen ZahIwort 18,84. - Zum altfriesischen Vokalismus 19,171. - Zu IF. 20, $361 \mathrm{ff}$. 22,331. - Za germanischen $\bar{e}^{2}, \bar{e}^{1} 23,92$. - Zu den sogen. reduplizierenden Präterita im Germanischen 23,103. - Zur pronominalen Flexion im Altgermanischen 26,174. - Zur germanischen Grammatik (1. Zum wg. hic-Pronomen. 2. Weiteres zum Sieversschen Gesetz über den konsonantischen Auslaut im Gotischen. 3. Zu urwg. $-\bar{a}-,-\hat{a}-(?)$ in gedeckter Endsilbe. 4. Zum vorgerm. Übertritt 
der alten adj. Flexion in die pronominale und Verwandtes) 27,278.

Hentze, C. Aktionsart und Zeitstufe der Infinitive in den homerischen Gedichten 22, 267.

Herbig, G. Aktionsart und Zeitstufe 6,157. - Indogermanische Sprachwissenschaft und Etruskologie 26, 360. - Altitalische Verbalformen 32,71. - Etruskisches Latein 37, 163.

Hermann, E. Beiträge zu den idg. Hochzeitsgebräuchen 17,373. Zur kyprischen Silbenschrift 19, 240. - Der kyprische Genitivus Singularis auf $-\omega$ v 20,354. - Homerisch oũtes 23, 164. - Zur Behandlung der antispastischen Wörter im homerischen Epos 25,285. - Die Länge geschlossener Endsilben im Griechischen 28,299. Über die primären Interjektionen 31,24. - Ist das Junglakonische eine künstliche Sprache? 32,358. - Norh einmal das Jungla konische 33,433. - Über die Apokope der griechischen Präpositionen 34,338. - Graeca 35,164. - Die dorische Betonung 38, 148.

Hertel, J. Die angebliche Wortfamilie utkalay, utkalappay, utkalāpana 29,215. - rathaspŕś oder rathaspirh 31, 143.

Hess, J.J. Zur Aussprache des Griechischen (Griechische Umschriften demotischer Wörter) 6,123.

Hessen, H. Die konsonantische Flexion in den Mailänder Glossen $30,225$.

Hillebrandt, A. Wurzel asth im. Sanskrit 5,388.

Hirt, H. Vom schleifenden und gestoßenen Ton in den idg. Sprachen 1,1. 195. - Die Urheimat der Indogermanen 1,464. - Zur Endung des Gen. sing. der Pronomina 2, 130. - Gehören die Phryger und Thraker zu den Satem-od. CentumStämmen? 2,143 - Zu den slav. Auslautgesetzen 2.337. - Die Verwandtschaftsverhältnisse der Indogermanen 4,36. - Über die mit $-m$ - und - $b h$ - gebildeten Kasussuffixe 5, 251. - Der Ackerbau der Indogermanen 5,395. - Zu den germanischen Auslautsgesetzen 6, 47. - Akzentstudien (1. germ. got. $\not \bar{u}$ sund $)_{\text {) }}$ 6,344. -- Akzentstudien (2. Die $n$-Stämme des Germanischen. 3. Zum grammatischen Wechsel der o-Stämme. 4. Die Dehnstufe im Serbischen. 5. Zur Sonantentheorie) 7,111. - Griech.

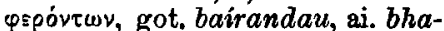
rantām 7,179. - Alkzentstudien (6. Die Abstufung zweisilbiger Stämme) 7,185. - Akzentstudien (7. Die thematischen Präsentien) 8,267. - Akzentstudien (9. Die Betonung des Vokativs im Indogermanischen. 10. Die Betonung der ersten Silbe im Ital. Kelt. und Germanischen) 9,284. - Akzentstudien (11. Die Stämme auf éi. 12. Zur Betonung des Preußischen. 13. Zur litauisch-slavischen Betonung. 14. Der idg. Ablaut $e-0$ ) 10,20. - Kleine grammatische Beiträge 12,195. - Zur Entstehung der griechischen Betonung 16,71. - Zur Bildung des griechischen Futurums 16,9\%. - Über den Ursprung der Verbalflexion im Indogermanischen 17,36. - Zur Verbalflexion 17,278. - Zur idg. Lautund Formenlehre (1. Zu den Gutturalreihen. 2. Zum n-Suffix im Lateinischen und Griechischen. 3. Zur Infinitivbildung im Griechischen. 4. Nochmals griech. ழEpóv$\tau \omega v)$ 17,388. - Zur Transskriptionsmisere 21,145. - Mistellen (1. er im Griechischen. 2. Idg. $\bar{e}^{m}$, $\bar{e}^{n}$ im Lateinischen. 3. Die Basis dõ 'geben'. 4. Metathesen. 5. Griech.

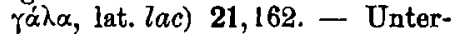
suchungen zur indogermanischen Altertumskunde 22, 55. - Zur Bildung auf $-\bar{\imath}$ im Indogermanischen 31,1. - Fragen des Vokalismus und der Stammbildung im Indogermanischen 32,209. - Zur Verbalflexion (1. Zum äol. Optativ. 2. Lat. nōvit $=$ ags. cnēow) 35,137 . - Zu den lepontischen und den thrakischen Inschriften 37,209. Grammatisches 37,217. - Etymologien 37, 227.

Hofmann, J. B. Znr lateinischen Wortforsehung und Syntax (1.Zur Etymologie von dis- und excidium. 2. Zur Bildung von domesticus, incommodesticus. 3. decem annos natus. 4. Zu passivischem amantis- 
simus, disiderantissimus, reverentissinius) 38,174. - Nochmals passivisches amantissimus 40,112.

Holthansen, F. Beiträge zur Erklärung und Textlkritik altenglischer Dichtungen 4,379. - Got. ahaks - lat. accipiter 5,274. Engl. culver - russ.gólubú 'Taube" 10,112. - Etymologien 14,339. Etymolngien 17, 293. - German. $a k$ 'sondern, aber' 17,458. - Etymologien I 20,316. - Etymologien II 25, 147.-Etymologien III 30,47. - Wortd eutungen 32,333. - Negation statt Vergleichungspartikel beim Komparativ 32,339. - Altenglische Etymologien 32, 340. - Etymologien 35, 132. Wortdeutungen 39,63.

Hoops, J. Alte $k$-Stämme unter den germanischen Baumnamen 14,478.

Horn, P. Zu den jüdisch-persischen Bibelübersetzungen 2,132. - Die Tieropfer im Avesta 2,365.

Horn, W. Angebliche Ellipse von lat.quam 17,100. - Zu IF. 39,72: an. be ocere 39,230 . - Zu IF. 39, 67: got. $w i t$ 'wir beide' 39,231 .

Hübschmann, H. Arisches und Armenisches 4,112. - Griech. po:\%ós und p:r.yós 11,200. - Die altarmenischen Ortsnamen 16,197.Armeniaca (l. Anl. $z$ im Armenischen. 2. Arm. zgam 'merke'. 3. Arm. nern 'Antichrist'. 4. Arm. ail = kypr. wìos. 5. Arm. gišer 'Schmelz'. 6. Arm. janjaxarit' 'scharlachrot'. 7. Kasusattraktion im Armenischen) 19,457 .

Hujer, O. Slav. domovz, dolovz 23, 152. - Slavische Miszellen 24,70. - Zur Deklination der Personalpronomina 30,49 .

Ijinskij, G. Aksl. ješa 'utinam' 28, 20.2. - Kirchenslavisch ovostb 'Frncht' 40, 144.

Ipsen, G. Lat. cuprum, griech. xúrpos und idg. *aios 39,232.

Jackson, A. V. W. Indo-Iranian Notes 25, 177.

Jacobi, H. Die Inversion von Subjekt und Prädikat im Indischen 5,335 . - Über den nominalen Stil des wissenschaftlichen Sanskrits 14,236. - Über eine neue Sandhiregel im Pāli und im Prakrit der
Jainas und über die Betonung in diesen Sprachen 31,211.

Janko, J. Über germanisch $\bar{e}^{2}$ und die sogen. reduplizierenden Präterita $20,229$.

Jellinek, M.H. Die Akzentabstufung eine Naturnotwendigkeit? $\mathbf{7 , 1 6 0}$. - Die Endung der 2. Person Plur. Praes. im Althochdeutschen 11, 197. - Beiträge zur Geschichte der Sprachwissenschaft 12, 158. Zur Geschichte einiger linguistischer Hypothesen 14,42. - Zur Geschichte einiger grammatischer Theorien und Begriffe 19,272.

Jiriczek, 0 . SCEPEN in,Caedmons Hymnus Hs. N. 30,279. - Tenuis für Media im Altenglischen 38,196. - Nachtrag zu S. 203 Z. 18 v.o. 38, 199 .

Johannson, A. Zu Noreens A bhandlung über Sprachrichtigkeit 1,232.

Johansson, K. F. Sanskritische Etymologien 2,1. -- Indische Miszellen 3,198. - Über skr. adbhyás, adbhis 4,134. - Indische Etymologien 8,160. - Arische Beiträge: 1. Zur Vertretung der idg. Dentalgeminaten im Arischen 14,265. 2. Über die idg. Verbindung Dental $+s(z)+$ Dental 19,119. - Zur mittelindischenGrammatik 25, 209.

Jokl, N. Über 'Etymologische Anarchie' und ihre Bekämpfung 27, 297. - Beiträge zur albanischen Grammatik (1.Zur Geschichte des alb. $t s$ in Erbwörtern. 2. Die Vertreibung von uralb. $z d$ aus idg. $s d(h), z d(h))$ 30,192. - Katun (Zur Geschichte tines Balkanwortes) 33,420. - Beiträge zur albanesischen Grammatik. (3. Der Akkusativ-Nominativ und der Geschlechtswechsel im Albanesischen) 36,98. - Beiträge zur albanischen Grammatik (4. Die Verbreitung der Dehnstufenbildung im Albanischen) 37,90.

Jolly, J. Sanskrit dohada, dvaihrdayya 10,213. - Lexikalisches aus dem Árthaśāstra 31,204.

Jostes, F. Idis 2,197.

Junker, H. Zu skr. mudrã 35,273.

Kahle, B. Altwestnordische Namenstudien 14,133.

Kalima, J. Nochmals russ, bygát 
'trocknen, dahinschwinden, verderben' 24,249 .

Kapteijn, J.M.N. DieÜbersetzungstechnik der gotischen Bibel in den Paulinischen Briefen 29,260.

Karsten, T. E Zur Frage nach den 'gotischen' Lehnwörtern im Finnischen 22,290. - Altdeutsche Kulturströmungen im Spiegel des finnischen Lehnworts 26,236 .

Kanffmann, F. Got.gawair bi31,321.

Keil, B. Griechische Dialektformen $36,236$.

Kent, R. G. The Oscan slingshot of Salpinum 32,196. - Lateinioches POVERO 'puero' 33,169.

Kern, J. H. Mist und die Wurzel migh 4,106. - Das Verbum āyūhati im Pāli 25,234. - Zum Verhältnis zwischen Betonung und Laut in niederländisch-limbürgischen Mundarten 26,258. - Deutung einer mißverstandenen Stelle im Mahāvastū 31,194.

Kieckers, F. Griechische Eigennamen auf -voos (-vo05) 23,353. Zum Gebrauch des Imperativus Aoristi und Präsentis 24,10. Prākritisches dinna und dinna ${ }^{\circ}$ gegeben'24, 289. - Das Eindringen der xo:vŕ in Kreta 27,72. - Zum altirischen $f-$ und $b$-Futur 27,325. - Die Stellung der Verba des Sagens in Schaltesätzen im Griechischen und in den verwandten Sprachen 30,145. - Zum Perfekt des Zustands im Griechischen 30 ,

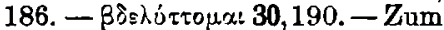
Akkusativus limitationis im Griechischen 30,361. - Zu den Schaltesätzen im Lateinischen, Romanischen und Neuhochdeutschen 32, 7 . - Verbalformen 32,87. - Zur 2. sing. des aktiven Imperfekts und zur 3. sing. des aktiven Imperativs des Präsens im Altirischen 34,408. - Zur oratio recta in den indogermanischen Sprachen 35,1. Armenisches (1. Zu den arm. Präsentien em 'ich bin' und berem 'ich trage'. 2. Zum arm. Aorist) 35,108. - pivrsios und ähnliches 35,289. - Mittelirisches clóthi 'Nägel' 35,341. - Zur oratio recta in den indogermanischen Sprachen II 36,1. - Griech. xtsivw xaivw 36,233. - Nochmals zum armenischen ç-Aorist 36,319 . $\mathrm{Zu}$ lat. $d \bar{a}$, dās 37,237. - Griech.

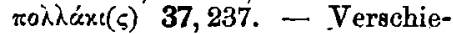
denes 38,209 - Zu mittelkymrisch heb 'sagte er' 39,123. - Zum Accusativus absolutus im Gotischen 39,125. - Zur 1. sing. ind. praes. auf $a \mathrm{im}$ Altbulgarischen 39,126. - Der elliptische Dual mit Ergänzungswort 39,207. - Imperativisches in indikativischer Bedeutung im Neuhochdeutschen 40,160. - Die direkte Rede als Objekt 40,248. - Zum passiven Imperativ im Lateinischen 40,250.

Kielhorn, J. Zu ai. tithi 20,228.

Kleinhans, E. Aw. hštua- 'sextus' 3,303.

Klnge, Fr. Germanisches 4,309. Vokativformen im Altenglischen? 6, 341. - Zum altpreußischen Wortschatz 21,358. - Griech. ठ̊́์ - Toptrios 39,129.

Knauer, F. Der russische Nationalname und die indogermanische Urheimat 31, 67.--ZurRusb-Frage 33, 394 .

Kock, A. Zum Wechsel $\bar{u}: \bar{o}$ in den an. Sprachen 2,332. - Zur Frage über den $w$-Umlaut, sowie über den Verlust des 20 in den altnordischen Sprachen 5,153. - Etymologisch-mythologische Untersuchungen 10,90. - Ein Beitrag zur gotischen Lautlehre 30,244. - Zur Frage nach dem Suffix der Participia Passivi altnordischer starker Verba 33,337.

Kögel, R. Zur altsächsischen Grammatik 3,276. - Germanische Etymologien 4,312.

Köhm, J. Der ursprüngliche Sinn von animum despondere und die zugrunde liegende Vorstellung $31,286$.

Kopucz, J. Die lateinischen Infinitive auf -ier 12,23.

Kossinna, G. Arminius deutsch? 2,174. - Die ethnologische Stellung der Ostgermanen 7,276.

Krěek, F. Talokus bei Bretkun 40,160 .

Krebs, H. Altpreufisch Mixskai 22,336 .

Krumbacher, K. хгท் $\omega_{\rho}$. Ein lexikographischer Versuch 25,393. 
Kryński, M. Z. de. Vieux-slave prégynja 29,227.

Lagercrantz, 0. Zwei griechische Fremdwörter 25,363.

Lambertz, M. Die hypothetische Periode im Albanischen 34,44

Lehmann, W. Ahd. hart, hartinheuui $21,192$.

Leskien, A. Die slavische Lautververbindung $j i$ 10,259. - Litauisches zavéti 'zaubern' 13,117. Schallnachahmungen und Schallverba im Litauischen 13,165. Litauisches esculus 'Buche'? 13, 279. - Litauische Partikeln und Konjunktionen 14,89. - Aksl. ojb 17,491. - Das Slavische in dem Etymologischen Wörterbuch der griechischen Sprache von Prellwitz 19,202. - Litauisches mozóti, màstëgriti 19,209. - Altkirchenslavisches ojbminz 19,398. - Altkirchenslavisches prégynja 21,197. - Über slavisches $o$ in Endsilben 21,335. - Zu IF. 21, 196; altkirchenslavisches ojzminz 21,338 . - Zur Frage der Einführung einer künstlichen internationalen Hilfssprache 22,389. - Zur Entstehung der exozentrischen Nominalkomposita 23, 204. - Litauische Personennamen 26,325. - Zur litauischen Wortkunde 28, 134. Über kirchenslavisches (altrussisches) serša 'Hornis" 28,137. $\mathrm{Zu}$ den litauischen Personennamen IF.26,325 28,390. - ZurTechnik der serbokroatischen Volkspoesie 31,413. - Litauisches iksauti 32, 205. - Die litauischen zweistämmigen Personennamen 34,296. Litauisches vẽlès 34,333, - Litauisches kiekolika 34, 336 .

Leumann, E. Einiges über Komposita 8, 297.

Leumann, M. Avestisch srifa- 39, 209. - Zur lateinischen Sprachgeschichte (1. Passivisches amantissimus. 2. ossua. 3. Lat. eei $>i \bar{i}$ ) 39,209. - Das lat. Suffix -aneus 40.116 .

Lewy, E. Etymologien 32,158. Preußisches 32,160.

Lewy, H. Kyprisches 1,506. Griechische Etymologien 2,445.

Lidén, E. Altenglische Miszellen 18,407. - Baumnamen und Ver- wandtes 18,485. - Zur iranischen Etymologie 19,316. - Zur germanischen Wortgeschichte 19,335. - Neue altenglische Miszellen 19,359 .

Liebich, B. Das Datum des Kalidasa 31, 198 .

Lindroth, H. Beiträge zur altnordischen Lautlehre. (I. Die sogen. Sonantisierungsfrage. U. Zum älteren $u$-Umlaut. III Zum älteren $i$-Umlaut) 29,129. - Über den Umlaut in den altnordischen $j a-$ Stämmen 35, 293.

Littmann, E. Parallelen zu der ' Verbindung von Ganzem und Teil' 35, 244.

Loch, E. Elliptisches $\tau \alpha \tilde{\tau} \tau \alpha$ in Grabinschriften $33,128$.

Loewe, R. Gotisch und Alanisch 3,146. - Das schwache Präteritum des Germanischen 4,365. Nochmals das schwache Präteritum im Germanischen 8,254. - Relative Chronologie der germanischen Tenuisverschiebungen 10,77. Die Krimgotenfrage 13,1. - Der Goldring von Pietroassa 26,203.

Loewenthal, J. Anord.Loki 29, 113.

Lorentz, Fr. Griech. Doōjos 5, 348. - Zu den germanischen Auslautgesetzen 5,380. - $\mathrm{Zu}$ den idg. to-Präsentien 8,68.

Haass, E. "I $p: s$ 1,157.

Marstrander, C. Etymologische Miszellen 20,346. - Germ. rukkan22,332. - Das Futurum von air. agid und der Indikativ von era 38,194 .

Meillet, A. Etymologies 5,328. A propos du groupe -ns- 10,61. - Les nominatifs sanskrits en - $t$ 18,417. - De l'accentuation des préverbes 21,339. - Sur le prétérito-présent got. lais 26,200. Des consonnes intervocaliques on védique 31,120 .

Meister, R. Zu den Regeln der kyprischen Silbenschrift 4,175. Die äoliscben Demonstrativa 8ys, $z_{v l}, z_{v v}$ und die Partikel ve (ve) im Phrygischen 25,312.

Meister, K. Arkadische Formen in der Xuthiasinschrift 18,77. - Der syntaktische Gebrauch des Genetivs in den kretischen Dialekt- 
inschriften 18,133. -- Altes Vulgärlatein 26,69 .

Heltzer, $K$. Vermeintliche Perfektivierung durch präpositionale $\mathrm{Zu}$ sammensetzung im Griechischen 12,319. - Zur Lehre von den Aktionen bes. im Griechischen 17, 187. - Gibt es ein rein präsentisches Perfekt im Griechischen? 25,338. - Nochmals das reine Perfekt 28,120.

Yeringer, $R$. Wörter und Sachen I 16,101. - Wörter und Sachen II 17,100. - Wörter und Sachen III 18.204. - Wörter und Sachen IV 19,401. - Wörter und Sachen V 21, 277.

Heyer, G. Etymologisches 1, 319. Von wem stammt die Bezeichnung Indogermanen? 2,125. - Lat. ligula 2,368. - Ngr. ăuı̌ 2,370.

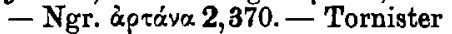
2,441. - Neugriechische Etymologien 3,63. - Zur Geschichte des Wortes Samstag 4,3:6. - Zum idg. $\bar{e}$-Perfektum 5,180. - Etymologisches aus den Balkansprachen 6,104 .

Meyer, R. M. Künstliche Sprachen I 12,33. - II 12,242. - Zur Geschichte einiger linguistischer $\mathrm{Hy}$ pothesen 13,126. - Die germanische Sprachbewegung 22,116. - Verba pluralia tantum 24,279.

Hoyer, K.H. Lat. habëre, got.haban und Verwandtes 35,224.

Meyer-Lübke, W. Der intensive Infinitiv im Litauischen und Russischen 14,114.

Mlchel, $\mathrm{K}$. Neupers. $m \bar{e} z \quad 30,448$.

Michels, V. Metathesis im Indogermanischen 4,58. - "Vgl. Wundt" 7,163. - Zur germanischen Lautverschiebung 14,224.

Michelson, T. The Indic ' root' khya in Pali and Prakrit 19,210. Pāli and Prākrit lexicographical notes 23,127. - Notes on the Pillar-Edicts of Asoka 23,218. The Etymology of the Girnār word Petenika- 24,52. - The alleged word adhigicya in the Bhabra Edict of Asoka 27,194. - Note on Pāli brahmun $\bar{a}, r \bar{a} j \bar{u} b h i$ 27,296. - Note on Old Russian krznuti, Pāli kināti 28,263. - The alleged Asokan word lukșa- 28,204. -
The alleged change of Indo-European $t s t(h)$ to $s t(h) 29,221$.

rikrola, J. J. Zum Wechsel von $b$ und $f$ im Germanischen 6, 311 . Slavica 6.349. - Slavica II 8, 302. - Woher lit. iau und slav. ju? 16,95. - Zur slavischen Etymologie 23,120. - Zwei slavische Etymologien 26,295.

Miller, Ws. Beiträge zur ossetischen Etymologie 21,323.

Miodoński, A. Auiare 13,142.

Mladenov, St. Etymologisches 35, 134. - Alb. buta und arm. but 38,169 .

IIogk, E. Die Inversion von Subjekt und Prädikat in den nordischen Sprachen 4,388. - Die Halbvokale $i$ und $u$ in der isländischen Literatursprache 26,209.

Möller, H. Hochton nach Auftakt 40,169 .

IIuch, R. Ister und Isar 8,287.

Iühlenbach, $K$. Über die vermeintlichen Genitive oder Ablative auf $-\dot{i}-u$ im Lettischen 13,220. Nachträge dazu 13,261. - Das Suffix -uma- im Lettischen 17,402.

Ytiller, G. H. Das Genus der 1ndogermanen und seine ursprüngliche Bedeutung 8, 304.

Muller, F. Zur Wortbetonung in den oskisch-umbrischen Dialekten 37, 187. - Lat. barba. (Zur Hauchdissimilation) 39,172 .

Neckol, G. Zu R. Meringers Ableitung von got. labōn 17,175. ExozentrischeKomposition 19,249. - Zum Instrumentalis 21,182. Die dreisilbigen Akzenttypen des Germanischen 40,123.

Nehring, W. Bemerkungen zu den $z$-Lauten im Slavischen, vornehmlich im Altslovenischen 4,397.

Nehring, A. Die Seele als Wasserblase 40,100 .

Niedermann, M. Studien zur Geschichte der lateinischen Wortbildung 10,221. - Etymologische Forschungen (Erster Teil): (A. Namen von Werkzeugen und Geräten. B. Baumnamen) 15,104. - Zur griechischen und lateinischen Wortkunde 26,43. - Zur indogermanischen Wortforschung 37,145 .

Noreen, A. Über Sprachrichtigkeit 
(für deutscheLehrer bearbeitet von A. Johannson) 1,95. - Etymologisches 4,320. - Suffixablaut im Altnordischen 14,396. - Ein Paar altnordische Seenamen 26,222.

Oertel, H. Über grammatische Perseverationserscheinungen 31,49 .

Oldenberg, H. Rgveda 10,13; 31,126.

Olsen, M. Zur thrakischen Inschrift von Ezerovo 38, 166. - Phrygisch

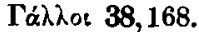

Osthoff, H. Labiovelare Media und Media aspirata im Keltischen 4, 264. - Griechische und lateinische Wortdeutungen 5,275.- Air. uan, ags. éanian : griech. ¿̊uvós 5,324. - Griechische und lateinische Wortdeutungen. Zweite Reihe 6, 1. - Griechische und lateinischo Wortdeutungen. Dritte Reihe 8,1. - Nachträgliches über lat. queo 9,179. - Griechisehe und lateinisehe Wortdeutungen. Vierte Reihe 19,217. — Gab es einen Instr. Sing. anf -mi im Germanischen? 20, 163. - Zur Entlabialisierung der Labialvelare im Keltischen 27,161.

Osten-Sacken, W. v. d. Zur slavischen Wortkunde 22, 312. - Nachträge dazu 22,340. - Etymologien 23,376. - Etymologien 24,238. -Zur Entwicklungsgeschichte der Nomina auf slavisch-sba, litauisch $-y b a s-y ́ b a-\tilde{y} b \dot{e}$, lettisch -ība 26, 307. - Etymologien 28,139. Die Ausbreitungstendenzen der Abstrakta auf lit-estis, - astis, lett. -ests, -asts, -estiba, astība 28,407. - Die Bedeutungssphäre der Eigenschaftsabstrakta auf slav. $-o b a$ 28,416. - Berichtigungen und Ergänzungen zu Waldes Iateinischem etymologischem Wörterbuch, 2. Auflage, aus dem Gebiet der Slavistik und Lituanistik 33, 181. - Zu Hirts Erklärung der indogermanischen es-Stämmo 34, 249. - Das litauische langvokalische Präteritum in seinem Verhältnis zum Infinitiv und Präsens 40,145. - Litauischer Vokalismus im Inlaut und Auslaut 40,251.

Otto, W. Über die lateinischen Wörter auf $-\bar{\imath} c a,-\bar{\imath} c u s,-\bar{\imath} c i u s,-\bar{x}$ and Verwandtes 15,9.

Patrubany, L. v. Idg. *ब|ozgh im Ar- menischen 13,124. - Armeniaca 13,163. - Zur armenischen Wortforschung 14,54. - Lituanica 32, 326.

Panl, H. Got. ai vor $r$ 4,334. Das Wesen der Wortzusammensetzung 14,251.

Pedersen, H. Das Präsensinfix n 2,285. - Das idg. $s$ im Slavischen 5, 33. - Die idg.-semitische Hypothese nnd die idg. Lautlehre 22, 341. - Zum slavischen $z$ 26,292.

Persson, P. Über den demonstrativen Pronominalstamm no- neund Verwandtes 2,199. - Zur lateinischen Grammatik und Wortkunde 26,60. - Etymologien 35, 149.

Petersen, W. Der Ursprung der Exozentrika 34, 254.

Petersson, H. Etymologische Beiträge 20,367. - Die indogermanischen Wörter für Milz 23, 158. Got. ibuks 23, 160. - Etymologien 23,384. - Einige Fälle von $\mathrm{Na}$ salinfigierung 24,38. - Zur indogermanischen Wortforschung 24. 250. - Die altindischen Wörter auf -amba-34,222. - Idg. * klonniund * $q o \hat{k s o-(-a) ~ 35,269 .}$

Planta, R. v. Eine dritte oskische Bleitafel 2,435. - Eine neue oskische Inschrift aus Capua 4, 258. - Nachträge zur Sammlung oskisch-umbrischer Inschriften 8, 315.

Pokorny, J. Keltisches (1. Idg. $\bar{e}$ im Keltischen. 2. Dehnstufige reduplizierte Aoristformen im Altirischen) 35, 172. - Zum altirischen Verbum (1.ZurStammbildung des redupl. Prät. 2. Zum Vokalismus von -icc-) 35, 336. - Zur Deutung des Futurums von altirisch agid 38, 115. - Die Lautgruppe ov im Gallobritischen 38,190. - Die Herkunft des irischen Artikels $39,217$.

Pokrowskij, M. Lateinische Zusammenrüickungen 26,100. - Zur lateinischen Nominalkomposition 31, 282 .

Pollak, H. W. Zur exozentrischen Komposition 30,55. - Zur Stellung des Attributs im Urgermanischen. (Ein Beitrag zur Geschichte des suffigierten Artikels 
im Altnordischen und der germanischen Kasuskomposita) $\mathbf{3 0 , 2 8 3 . ~}$ - Nochmals zur Stellung des Attributs im Urgermanischen 30,390.

Porzesinski, V. Der Dativ Sing. der $i$-Stämme im Litauischen 31,423.

Postgate, J. P. The Future Infinitive Active in Latin 4,252. Three Latin Etymologica 26,115.

Preusler, W. Zu A. Hillebrandt, Der freiwillige Feuertod in Indien 39, 208 .

Prokosch, E. Die Stabilität des germanischen Konsonantensystems 33, 377.

Purdie, E. The Perfective 'Aktionsart' in Polybius 9,63.

Reckendorf, $\mathbf{H}$. Zur allgemeinen Syntax 10, 167.

Reichelt, H. Der steinerne Himmel 32,23. - Die Labiovelare 40,40.

Richter, 0 . Die unechten Nominalkomposita des Altindischen und Altiranischen. Erster Teil 9,1. Zweiter Teil 9,183.

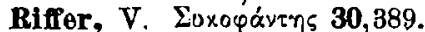

Rodenbasch, E. Bemerkungen zur Satzlehre (1. Die Agensform als Subjekt. 2. Die sogenannten unvollständigen Sätze) 19,254 . Die syntaktiche Entwicklung des lateinischen Conjunktivus Imperfekti 20,258. - Beiträge zur Geschichte der griechischen Aktionsarten 21,116. - Zur Bedeutungsentwicklung des griechischen Perfekts 22, 323. - Präsensstamm und perfektive Aktionsart 22,402. -Die temporale Geltung des Part. Aor. im Griechischen 24,56. Zur Frage nach dem Alters- und Verwandtschaftsverhältnis zwischen Optativ und Potentialis 24, 181. - Präsentia in perfektischer Bedentung 28,252.

Rozwadowski, J. v. Der demonstrative Pronominalstamm ol- 3,264. - Zu dem slavischen Iterativa 4,406. - Slav. pizdá 'cunnus, vulva' 5,353. - Der litauische Akzent in der "Universitas linguarum Litvaniae" 7,233. - Ein quantitatives Gesetz der Sprachentwicklung 25, 38 .

Rntz, 0. Neues über den Zusammenhang zwischen Dichtung und Stimmqualität 28,301.
Saussure, F. de. Sur le nom. plur. et le gén. sing. de la déclinaison consonantique en lituanien $\mathbf{4 , 4 5 6}$.

Scheftelowitz, J. Das Schicksal der idg. Lautgruppe $z g$ 30,133.

Schischmánov, J.D. Der Lenorenstoff in der bulgarischen Volkspoesie 4,419.

Schlachter, L. Statistische Untersuchungen über den Gebrauch der Tempora und Modi bei einzelnen griechischen Schriftstellern (I. Homer) 22, 202. - Fortsetzung (II. Herodot) 23,165. - Fortsetzung (III. Thukydides) 24,189.

Schmidt, B. Ganz 33, 313.

Schmidt, R. Zur keltischen Grammatik 1,43 (I. Neuir. cúig 'fünf'. caoga' fünfzig' und Verwandtes 43. - II. Ưber bretonisches - $m p$ im Verbal- u. Pronominalsysteme 50. - III. Über die Vertretung von idg. Nasalis sonans im Irischen und Verwandtes 59).

Schmidt-Wartenberg, H. Zur Physiologie des litauischen Akzents 7,211. - Phonetische Untersuchungen zum lettischen Akzent 10,117 .

Schmitt, J. Myrolog oder Moiro$\log 12,6$.

Schöll, F. Zur lateinischen Wortforschung (1. senecta - iuventa. 2. Zwei angeblich spanisch-lateinische Wörter) 31,309.

Schrader, 0 . Über Bezeichnungen der Heiratsverwandtschaft bei den idg. Völkern 17,11. - Der Hammelsonntag. Eine Reisestudie aus dem Gouvernement Olonez 26, 297.

Schröder, E. Die 2. Pers. Sing. Perf. st. Flexion im Westgermanischen $39,224$.

Schröder, H. Nhd. lehne, lenne 'Spitzahorn, acer platanoides L.' 17,316. - Zur Etymologie von ahd, scarph, scarf; anord. snarpr; ahd. sarf, sarph; (saro; sërawên) 17,459 . - Die germ. Wurzeln stelund ster- und ihre durch $p, k, t$ erweiterten Formen 18,509. Etymologisches 22,193. - Nochmals nd. man 'nur' 24,25. - Zu IF $+22,193$ ff. 24,55 .

Schröder, L. v. Der siebente Aditya 31,178.

Schuchardt, H. Über den aktivi- 
schen und passivischen Charakter des Transitivs 18,528.

Schütte, G. Über die alte politische Geographie der nichtklassischen Völker Europas 15,211. - Die Schöpfungssage in Deutschland und im Norden 17,444.

Schweizer, E. Griechische Miszellen 10,204.

Schwering, W. Lateiniseh Aiax, Aiācis 30,220. - Nachträgliches zu lat. $A i \vec{a} x$ 32,364. - Deus und divus 34, 1. - Die Entstehung des Wortes tragicomoedia 37, 139 ,

Schwyzer, E. Varia zur griechischen und lateinischen Grammatik 14,24. - Nhd. Rahm: jgav. raorna 21,180. - Syutaktisches (1. Zum Genitiv bei es-. Ai. ca 'wenn') 23,162, - Etymologisches 23, 307. - Die Demonstrativpronomina des Schweizerdeutschen 26,283. Osk. ist 27,293. - Haplologie im Satzzusammenhang 28,300. - Zur griechischen Etymologie 30,430. - Z.wei Vermutungen za Homer (1. 'Die Insel der Morgenröte'.

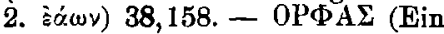
Beitrag zur griechischen Dialektologie und zur delphischen Topographie) 38,161. - Erhaltender Einfluß nicht-idg. Sprachen auf dio idg. Deklination? 38,165. - Nachtrag dazu 39,130.

Sievers, E. Germanisch $l l$ aus $d l$ 4,335. - Zu den angelsächsischen Diphthongen 14,32. - Nachtrag dazu 15,336. - Angelsächsisch weeriz 'verflucht' 26,225. - Zur nordischenVerbalnegation 31,335 . Ags. zéazl 34,337.

Sigwart, G. Darius oder Dareus? $35,289$.

Skutsch, F. Zu den etruskischen Zahlwörtern 5,256. - Latina 14, 485.

Smith, J. A. Indoeuropean -ssbetween rowels 12,4 .

Solmsen, F. $\Delta$ áx Inf. praes. act. und die $i-\mathrm{Di}$ phthonge in wortschließenden Silben im Lateinischen 4,240. Got. ale w 5,344. - Lakonisch Eipๆv 7, 37. - ZweiverdunkelteZu-

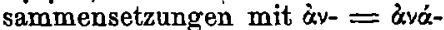
13,132. - $\Delta: \zeta \eta_{1} \mu \alpha !$ si $\zeta_{0 \mu \alpha}$ und d: $\zeta \omega ~ 14,426$. - Zur lateinischen

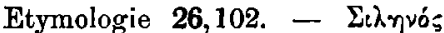

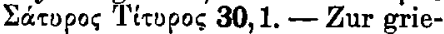
chischen Wortforschung 31,448 . Sommer, F. Lateinisch mille 10, 216. - Die Komparationssuffixe im Lateinischen. I. Die komparativischen Suffixe 11,1. - Das slavische Iterativ-Suffix -vati 11, 202. - Die Komparationssuffixe im Lateinischen. II. Die superlativischen Suffixe 11,205. - Erwiderung 11,323. - Lateiniacher Vokalumlaut in haupttonigen Silben 11,325. - Lateinische Miszellen 14,233. - Lat. alis und aliquis 24,17. - Der Dativus pluralis der 3. Deklination im Nordwestgriechischen 25,289. - Zum indogermanischen Personalpronomen 30,393. - Zur deutschen Wortforschung (1.Dachs. 2. eben. 3. Gaul. 4. keusch. 5. Quarz) 31, 359. - Das Femininum der $u$ und $i$-Adjektiva im Rgveda und im Altiranischen 36,165. - Zur Syntax des slavischen GenitivAkkusativ bei belebten Wesen 36, 3)2. - Oskisch iiv 38, 171.

Sonnenburg, P. E. Zur Ableitung von calefacio und calebam $12,386$.

Speyer, J.S. Ein syntaktisches Klee: blatt (1. Prädikativer Nominativ im klassischen Sanskrit, 2. Bemerkungen zu dem sogenannten periphrastischen Futurum des Sanskrit. 3. Lat. agone? und Verwandtes) 31, 108 .

Spitzer, L. Albanisches (1. Zwei Tiernamen. 2. 'Hiatustilgendes' * im Albanischen? 3. Ist lat. $\bar{e}$ im Albanischen zu ie geworden?) $39,105$.

Stackelberg, R. v. Persische Miszellen 4,147.

Stokes, Wh. On the assimilation of pretonic $n$ in Celtic suffixes 2,167. - Irish Etymologies 12, 185. - $s$-Presents in Irish 22,335. - Irish Etymologies 26,139.

Stolz, Fr. Lat. strufertārius 1,332. - Zum Konjunktiv des griechischen sigmatischen Aorists 2,154. - Zur Chronologie der Iateinischen Lautgesetze 4, ¿33. - Glôria 10, 70. - Zur lateinischen Sprachgeschichte 13,95. - Zur griechischen und zur lateinischen Sprach- 
geschichte 14,15. - Lateinisch püsus, pütus und Verwandtes 15, 63. - Zum lateinischen Wortschatz 17,85. - Lateinische Miszelle 17,441. -- Nachtrag zu 17, 90 f. 18, 132. - Neue Beiträge zur lateinischen Sprachgeschichte und Lautlehre 18,439. - Laverna 22, 242. - Dio Flexion von lavere und lavāre 26,119.

Strachan, J. Lat. perendie 1,500. - Keltische Etymologien 2,869. -The Nominative Plural of Neuter $u$-stems in Celtic 10,76.

Streitberg, W. Betonte Nasalis sonans 1,82. - Der Genitiv Pluralis und die baltisch-slavischen Auslautgesetze 1,259. - Anord. tyggja und Verwandtes 1,513. Vokalkürzung im Baltischen 2,415 . - Vokaldehnung vor tautosyllabischem -ns im Baltischen 3,148. - Die Entstehung der Dehnstufe 3,305. - Ost- und Westgoten 4, 300. - Mattium, Maltiacus 5,87. - Akzentfragen 5,231. - Zum Zablwort 5,372. - Griech. 'A ${ }^{2}$ acoi ägypt. 'Alkajwaša 6,134. - Zur gerraanischen Grammatik 6,140. - Die griechischen Lokative auf દí 6,339. - Urgerm. zin 7, 177. Schleichers Auffassung von der Stellung der Sprachwissenschaft 7,360. - Germanisches 14,490. - Gotica (1. $z b d$ im Auslaut. 2. Got. ni waihts. 3. Spirantendissimilation und Kompositum) 18,383. - Gotisch twa pousundja 18,421. - Zur Flexion des gotischen Adjektivs 19,214. $\rightarrow$ Got. sunnin 19,391. - Zum gotischen Perfektiv 21,193. - Gotisch $d u$ gunnun wisan 22,307. - Gotisch fraujinond frauja 23,117. - Go.

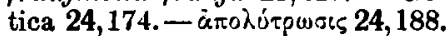
- Zum Perfektiv 24,311. - Kant und die Sprachwissenschaft. Eine historische Skizze 26,382. - Gotica 27,151. - Gotica (1. sabbato. 2. Abeileni. 3. gadaila) 31,323 . - Zu GraBmanns Gresetz 34,366. - Zur Geschichte der Sprachwissenschaft (1.Persisch und Deutsch. 2. Der Wandsbecker Bote als Sanskritist. 8. Agglutination. 4. Lachmanns Gesetz) 35, 182. - Die Bedeutung des Suffixes -ter 35,196.
- Zum schwachen Präteritum 35, 197. - Zu IF. $39,13039,230$.

Strömberg, E. Die Entstehung von $-\bar{o} z-$ in der germ. Komparation 20,361.

Stitterlin, L. Etymologisches Allerlei 4,92. - Zwei Beispiele der Aussprache des heutigen Englisch 14,459. - Die Denominativverba im Altindischen 19,480. - Der Schwund von idg. $i$ und $u 25,51$. - rotundus und die lat. Gerundialformen 27,118. - Aus meinem etymologischen Sammelkasten I. 29,122 .

Srerdrup, J. Über die Lautverbindung $\chi s$ im Germanischen, besonders im Altnordischen 35,149.

Szilasi, M. Veneres Cupidinesque 17,442 .

Tamm, Fr. Über einige slavische Wörter im Schwedischen 4,395.

Thomson, A. Die Eigentöne der Sprachlaute und ihre praktische Verwendung 24,1. - Beiträge zur Kasuslehre (I. Über den GenitivAkkusativ im Slavischen) 24,293. - Beiträge zur Kasuslehre (II. Der Genitiv bei Massenbezeichnungen besondersim Russischen) 28,107.Beiträge zur Kasuslehre (III. Zur Genitivrektion des Verbums im Baltischslavischen) 29,249. - Beiträge zur Kasuslehre (IV. Ü̉ber die Neubildungen des Akkusativs) 30,65 .

Thumb, A. Beiträge zur neugriechischen Dialektkunde (I. Der Dialekt von Amorgos) 2,65. Die ethnographische Stellung der Zakonen 4,195. - Beiträge zur neugriech. Dialektkundo (I. Der Dialekt von Amorgos. Fortsetzung) 7,1. - Zur Aussprache des Griechischen 8,188. - Ein neuer inschriftlicher Beleg für aspiriertes $\hat{\delta}$ im Anlaut? 8,227. - Zur Geschichte der griech. Digamma 9, 294. - Alt- und Neugriechische Miszellen 14,343. - Psychologische Studien über die sprachlichen Analogiebildungen 22,1. - Altgriechische Elemente des Albanesischen 26.1. - Zur Aktionsart der mit Präpositionen zusammengesetzten Verba im Griechischen 27,195. - Über die Behandlung 
der Lautgruppe $-\sigma \vartheta-$ in den nordwestgriechischen Dialekten 31 ,292. Ist das Junglakonische eine künstliche Sprache? 33,294. - Antwort 33,434 .

Thurneysen, $R$. Das sog. Präsens der Gewohnheit im Irischen 1,329. - Der irische Imperativ auf the 1,460. - 1. Der Präsenstypus

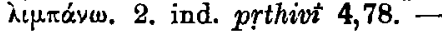
Spirantenwechsel im Gotischen 8,208. - Etymologien 14,127. Etymologisches und Grammatisches 21,175. - Die irische Personalendung -enn, ann 26,131. Berichtigung dazu 27,160. - Zur Wortschöpfung im Lateinischen (I. Purgare. II lēvigare - lěvigare) 31,276. - Zum indogermanischen und griechischen Futurum 38, 143 .

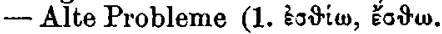

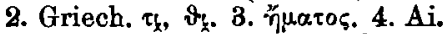
dadáu. 5. Der ai. Dat. auf āya. 6. Lat. paullisper. 7. Lat. hic, iste. 8. Lat. disco, mitto 9. Zur lat. V. Dekl. 10. permities) 39, 189.

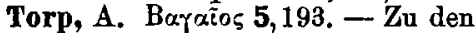
messapischen Inschriften 5,195 .

Trantmann, R. Ahd. zueio 'zu zweien' 38,199 .

Treimer, K. Der albanische Nationalname, gegisch skiüp, toskisch $s k^{\prime}$ ip 35, 135 .

Uhlenbeck, C.C. Agens und Patiens im Kasussystem der indogermanischen Sprachen 12,170. - Die Vertretung der Tenues aspiratae im Lateinischen 13,213. - Nachtrag zu 12,170 13,219. - Die Vertretung der Tenues aspiratae im Slavischen 17,93. - Nachtrag dazu 17, 176. - Eine baskische Parallele 17,436. - Eine dex ÉxaxosZusammensetzung im Baskischen 21,197. - Etymologika 25, 143. - Baskisch und Indogermanisch 33,171.

Vasmer, M. Die Flexion von altbulg. kyjz 'qui' 40,139.

Vendryes, J. A propos de la flexion du présent irlandais tíagu 'je vais" 26, 134.

Vondrák, W. Zur Erkläruug des aksl. Dat. Sing pati, Rosti 10,113.

Wackernagel, J. UUber ein Gesetz der idg. Wortstellung 1,333. Griech. $\pi$ útp 2,149. - Griech. ктะрео̃̃อย 2,151. - Zur griechischen Nominalflexion 14,367. Zur Umschreibung der arischen Sprachen 22, 310. - Attische Vorstufen des Itazismus 25,326. Lateinisch-Griechisches (1. Dissimilationserscheinungen. 2. $o p$ tare. 3. parabola. 4. quia. 5. Die Genetive auf -ius) 31,251. - Zu altir. fitir 39, 220. - Zu der altpersischen Stelle in Aristophanes' Acharnern 39,224.

Wadstein, E. Nordische Bildung mit dem Präfix ga- 5,1. - Raub, Robe und Verwandtes 14,402.

Walde, A. Zur Entwicklung von germ. ai im Friesischen 12,372. - Aspiratendissimilation im Lateinischen 19,98. - Zu den indogerm. Wörtern für ' Milz' 25,160. Odium und der Betrieb der lateinischen Etymologie 28,396. Nochmals odium 30,139. -. Lateinische Etymologien 39,74. Umbrisch purditom 39,216 .

Wegener, $\mathrm{Ph}$. Der Wortsatz 39,1. Weyman, C. Lat. oportunus 5,194. Wheeler, B. J. Greek Duals in $-\varepsilon$ 6,135 .

Whitney, W. D. Examples of sporadic and partial phonetio change in English 4,32.

Wiedemann, 0 . Got. hrot 1,194. Zur Gutturalfrage im Lateinischen 1,255. - Got. saíhan 1,257. Got. faírguni 1,436. - Gotische Etymologien 1,511.

Wilsdorf, $R$. Ein altlateinischer Dativ der fünften Deklination auf -iei 35,97.

Wijk, $\mathbf{N}$. v. Welchen Platz nehmen die griechischen Nomina auf -zús unter den nominalen Stammbildungsklassen des Indogermanischen ein? 17,296. - Die altitalischen Futura 17,465. - Zur Konjugation des Verbum substantivum 18,49. - Ags. cú, an. kÿr 19,393. - Zum indogermanischen Ablaut 20,332. - Germanisches (1. Germanisches $\vec{a}$ in auslautenden Silben. 2. An. tuau,

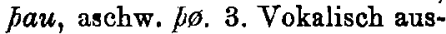
lautende Instrumentale von Pronominalstämmen) 22,2ล0. - Anlautendes idg. $d l$ - im Germanischen 23,366. - Ahd. deo, dio, blinto, 
alem. kebo 24, 28. - Germanische Etymologien 24,30. - Fortsetzung 24,230. - Eine polnisch-niederländische Parallele 26,275. - Germanische Etymologien 28, 121. Berichtigung zu IF. 24,236 ff. 28, 204. - Abg. inz, jedınz, otznqdъ 30,382. - Das indogermanische Wort für Ameise 33,367. - Das litauische langvokalische Präteritum 34,367. - Zur Etymologie einiger Wörter für 'leer' 35,265 . - Zum altbulgarischen $l_{6} 35,342$.

- Zu IF. 33,367 ff. 35,347.

Zum baltischen und slavischen Akzentverschiebungsgesetz $\mathbf{4 0 , 1}$. - Die serbokroatischen Präsentia von Präpositionalkomposita mit betonter Präposition 40,261. Die aus altem Akutus entstandenen sekundären slavischen Intonationen 40,275.

Wiklund, K. B. Die ältesten germanischen Lehnwörter in Finnischen 38,48 .

Windisch, E. Beiträge zur Etymologie und Bedeutungslehre $\mathbf{3 , 7 8}$. - Zu den irischen Zahlwörtern 4,294. - Pronumen infixum im Altirischen und im Rgveda 14,420. -Zum Pronomen infixum 15,126.

Wislicenus, $P$. Vokalunterströmungen 23, 271 .

Witte, K. Zur homerischen Sprachund Verstechnik 32,148.

Wollner, W. Einige Spuren des Einflusses der iranischen Heldensage auf die südslavische $\mathbf{4 , 4 4 8}$.

Wood, F.A. Phonetic Notes 13,119. - How are words related? 18,1 . - Rime-words and Rime-ideas 22, 133.

Leipzig.
Wunderlich, H. Gewand und Gewaete 14,406.

Wundt, W. Sprachwissenschaft und Völkerpsychologie 28,205.

Zachariae, Th. Sanskrit mutkala 30,366. - Sanskrit urvarita 'übrig' 32,341 .

Zimmermann, A. Die Endung -por in Gaipor, Lucipor usw. 15,121. - Etymologische Beiträge 15,123. - Zu mhd. enenkel 'Enkel '15, 339. - Zur Entwicklung des Suffixes -tor (-ter) im Lateinisehen 18,376. - Zur Etymologie von september, -bris 19,210. - Randbemerkungen zu ein paar Stellen von Brugmanns GrundriB $2,130,216$. - Noch einmal Aiax 32,202. - Zu IF. 30, 219 32, 204. - Ist dit Stadt Rom notwendig als eine Siedlung des Geschlechts der tuskischen rüma zu betrachten? 32,414, - Erwiderung (vgl. IF. 32,364) 33,435.

Zubatý, J. Baltische Miszellen (1. Über einige lit und lett. adverbiell gebrauchte Instrumentalbildungen) 3,119. - Baltisch $\theta$ Miszellen (2.-5.) 4,470. - $\mathrm{Zu}$ ai. krimis, Jat. vermis usw. 6,155. - Baltische Miszellen (6. Die Postpositionen -an -en und die litauisch-lettischen Lokale. 7. Zu den lettischen Genetiven auf $-\dot{u}$ -u) 6,269. - Baltische Miszellen (8. Zu lit. paskuĩ, pãskui usw.) 7, 182. - Baltische Miszellen (9. Der ursprachlishe Loc. Du. im Litauischen) 8,214. - Ai. tithi, tithih 'lunarer Tag' 19,370. - Haplologie im Satzzusammenhang 23, 161. - Zum Gebrauch von ved. viśvah̆ 'omnis' 25, 200 .

Walter Porzig. 